\title{
miRNA expression profile in primary gastric cancers and paired lymph node metastases indicates that miR-10a plays a role in metastasis from primary gastric cancer to lymph nodes
}

\author{
WEIDONG CHEN $^{1 *}$, ZHAOQING TANG $^{1 *}$, YIHONG SUN $^{1}$, YOUYOU ZHANG $^{2}$, XUEFEI WANG $^{1}$, \\ ZHENBIN SHEN $^{1}$, FENGLIN LIU ${ }^{1}$ and XINYU QIN ${ }^{1}$ \\ ${ }^{1}$ Department of General Surgery, Zhongshan Hospital, Fudan University, Shanghai; \\ ${ }^{2}$ Institutes of Biomedical Sciences, Fudan University, Shanghai, P.R. China
}

Received August 30, 2011; Accepted November 22, 2011

DOI: 10.3892/etm.2011.411

\begin{abstract}
The aim of this study was to identify and evaluate microRNAs (miRNAs) in gastric cancer lymph node metastasis. A miRNA array was used to compare the expression of miRNAs in primary gastric cancer and paired lymph node metastases. miRNAs found to be differentially expressed were validated in a cohort of primary gastric cancer tissues, and the relationship between expression and the clinicopathological characteristics of the specimens was analyzed. The expression level of miR-10a in a gastric mucosal cell line and three gastric cancer cell lines was also detected using qPCR. Moreover, the target genes for miR-10a were predicted using bioinformatic methods. Based on the results, four differentially expressed miRNAs were detected by the miRNA array. Compared with primary gastric cancer, lymph node metastases displayed downregulated expression of miR-24-1*, miR-510 and miR-1284, while the expression of miR-10a was upregulated. Consequently, analysis found that the expression of miR-10a was associated with lymph node metastasis $(\mathrm{P}=0.047)$, but was independent of the state of lymphatic invasion $(\mathrm{P}=0.169)$ in the cohort of primary gastric carcinoma. The expression of miR-10a was at least 10-fold higher in the three gastric cancer cell lines than in the gastric mucosal cell line. Two gastric cancer cell lines, which were established from lymph node metastasis, expressed higher miR-10a compared to the primary tumor origin cell line. Bioinformatic analysis demonstrated that the target genes of miR-10a are involved in multiple related pathways of tumorigenesis and metastasis. In conclusion, our study suggests that miR-10a is involved in the development of gastric cancer and lymph node metastasis, particularly in the latter process.
\end{abstract}

Correspondence to: Dr Yihong Sun, Department of General Surgery, Zhongshan Hospital, Fudan University, Shanghai 200032, P.R. China E-mail: yihongsun@medmail.com.cn

${ }^{*}$ Contributed equally

Key words: miR-10a, gastric cancer, lymph node metastasis

\section{Introduction}

Gastric cancer is one of the most common malignant tumors of the digestive system, and its early diagnosis rate is still low in China, where gastric cancer is typically diagnosed at a more advanced stage; metastasis is typically present at the time of diagnosis. However, conventional strategies based on radical surgery for the treatment of gastric cancer are not yet satisfactory. Therefore, investigation of the mechanisms of metastasis and recurrence of gastric cancer, and exploration of the methods for the effective prediction, prevention and treatment of tumor metastasis are attracting increased attention in both basic and clinical cancer research.

microRNAs (miRNAs), discovered in 1993, are a class of non-coding RNAs 19-25 nt in length that regulate gene expression at the post-transcriptional level $(1,2)$. These miRNAs regulate complicated cellular processes such as differentiation, proliferation and apoptosis (3) and are widely involved in tumorigenesis, tumor progression and metastasis (4,5). Recent research suggests that miRNAs are involved in tumor invasion and metastasis, in the context of breast cancer, colorectal cancer, esophageal squamous cell carcinoma, and pancreatic endocrine tumors $(6,7)$. Few studies have reported the role of miRNAs in gastric cancer owing to its relatively low incidence in Europe and America. Therefore, profiling should be performed in different types of gastric cancer to identify differentially expressed miRNAs. In the present study, we investigated differences in miRNA profiling between primary gastric cancer and paired lymph node metastasis, and then detected the differentially expressed miRNAs in 4 cell lines, 33 gastric cancer specimens and corresponding adjacent gastric mucosa tissue, in an effort to identify the miRNAs involved in gastric cancer lymph node metastasis. Furthermore, our data provide evidence regarding the effects of miRNAs on metastasis involved in the molecular pathogenesis of gastric cancer.

\section{Materials and methods}

Specimens

Specimens used in the miRNA array. Cases of full-thickness gastric wall with invasion and lymph nodes with tumor 
Table I. Data from the gastric cancer patients.

\begin{tabular}{lcccccccc}
\hline No. & Gender & Age & Tumor location & Type & Differentiation & $\mathrm{T}^{\mathrm{a}}$ & $\mathrm{N}^{\mathrm{a}}$ & \multicolumn{2}{c}{ Others } \\
\hline 1 & Male & 67 & Lower 1/3 & Adenocarcinoma & II & 3 & 3 & Lymphatic invasion \\
2 & Male & 54 & Mid 1/3 & Adenocarcinoma & II-III & 3 & 1 & Lymphatic invasion \\
3 & Male & 62 & Lower 1/3 & Adenocarcinoma & II & 3 & 2 & Unknown \\
4 & Male & 52 & Lower 1/3 & Adenocarcinoma & III & 3 & 1 & Neural invasion \\
5 & Male & 76 & Lower 1/3 & Adenocarcinoma & III & 3 & 3 & Neural invasion \\
\hline
\end{tabular}

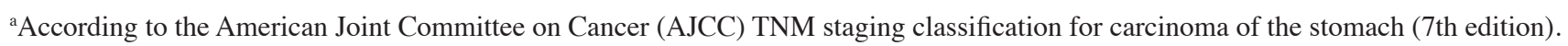

metastasis were assessed intraoperatively; specimens from the primary tumor were retained. The specimens were embedded in optimal cutting temperature compound (OCT) and stored at $-80^{\circ} \mathrm{C}$ until use. Experimental samples were selected to pathologically confirm that invasive adenocarcinoma of the stomach lesions was of full thickness and to verify the existence of lymph node metastasis. In total, 5 pairs of specimens (each of which included primary tumor and paired lymph node metastasis tissues) were selected, and their clinicopathological characteristics are summarized in Table I.

Specimens used for validation. Surgical specimens were obtained from 33 randomly selected patients with primary tumors among those who underwent D2 radical resection of gastric cancer at ZhongShan Hospital, Fudan University (Shanghai, China) from May, 2006 to December, 2007. All eligible cases had not received preoperative tumor-related treatment, were pathologically confirmed as gastric adenocarcinoma, and displayed full-thickness gastric wall invasion. Clinicopathological statistical data are shown in Table IV. Another 10 cases were randomly selected from the 33 cases mentioned above; normal gastric mucosa tissues were taken from the tumor marginal zone ( $>5 \mathrm{~cm}$ from the tumor). These samples were mixed in proper proportion after RNA extraction and used as the control group (indicated below with an N). Random sampling was performed with SPSS software. Ethical approval was obtained from the ZhongShan Hospital Research Ethics Committee.

Gastric cancer cell lines. Human gastric cancer cell lines, AGS and SGC7901, were purchased from the Cell Bank of the Chinese Academy of Sciences. The human gastric mucosal cell line GES-1 and moderately differentiated tubular adenocarcinoma cell line MKN-28 were a gift from the Institute of Digestive Disease, Shanghai Jiaotong University. All cells mentioned above were cultured in RPMI-1640 medium supplemented with $10 \%$ fetal bovine serum in $5 \% \mathrm{CO}_{2}$ at $37^{\circ} \mathrm{C}$.

\section{Methods}

Laser capture microdissection (LCM). The collected tissues embedded in OCT were cut in $8-\mu \mathrm{m}$ sections at $-20^{\circ} \mathrm{C}$, after being stained with hematoxylin and eosin (H\&E). The stained slides were placed into a Veritas Microdissection Instrument (Veritas, Mountain View, CA). Areas that were enriched in tumor cells (10 $\mathrm{mm}^{2} /$ slides) were selected and captured using ultraviolet laser cutting according to the manufacturer's recommended protocol. The tissue samples were immediately placed in a microcentrifuge tube containing lysis/binding buffer (Ambion, Inc., USA), which was vortex-mixed and stored upside down at $-20^{\circ} \mathrm{C}$. RNA concentration and integrity were determined by $\mathrm{UV}$ absorption and agarose gel electrophoresis, respectively.

miRNA array. miRCURY LNA ${ }^{\mathrm{TM}}$ microRNA arrays (Agilent Technologies, Santa Clara, CA), which contain more than 1,700 capture probes, were used to compare the expression profiles of 5 paired primary gastric cancer tissues and metastatic lymph nodes collected from the same case. A difference in expression was defined as a change of $>1.5$-fold in the corresponding level of miRNA detection between paired specimens. The Student's t-test was used to analyze whether there were significant differences between paired samples.

qPCR assessment of miRNA (8). miRNA genes were selected from the Sanger Center miRNA Registry (http://www. mirbase.org). The sequence-specific primers for miRNAs and endogenous control U6 snRNA were synthesized by Sangon (Shanghai, China) (Table II). The reverse-transcription reaction was carried out with miRNA-specific stem-loop RT primer and reverse transcriptase (Fermantas, USA) according to the manufacturer's instructions. Real-time quantification polymerase chain reaction (qPCR) analysis was carried out after cDNA was synthesized. Reaction conditions were as follows: $95^{\circ} \mathrm{C}$ for $10 \mathrm{~min}$; followed by 40 cycles of $95^{\circ} \mathrm{C}$ for $15 \mathrm{sec}, 60^{\circ} \mathrm{C}$ for $15 \mathrm{sec}$, and $72^{\circ} \mathrm{C}$ for $45 \mathrm{sec}$ for the amplification. The threshold cycle $(\mathrm{Ct})$ is defined as the fractional cycle number at which the fluorescence passes the fixed threshold. The gene expression $\mathrm{Ct}$ values of miRNAs from each sample were calculated by normalization to the internal control U6 snRNA. All experiments were repeated in triplicate.

Bioinformatic analysis of miRNA. miRGen (http:// www.diana.pcbi.upenn.edu/miRGen/v3/miRGen.html) was employed for the prediction of miR-10a target genes. Functional analysis of the predicted targets was performed using MAS3.0 Bioinformatics Resources (http://bioinfo.capitalbio.com/ $\operatorname{mas} 3 /)$.

Statistical analysis. miRNA expression in tumor samples was detected by real-time quantitative PCR and then subjected to $\mathrm{N}$ normalization. The relationship between changes in miRNA expression and clinicopathologic characteristics was analyzed using one-way ANOVA. Two-tailed $\mathrm{P}<0.05$ was judged to be significant. All data and statistics processing were performed with Excel 2007 (Microsoft, USA) and SPSS 16.0 (SPSS, Chicago, IL). 
Table II. Primer sequences used for miRNA expression analysis with gene name, sequence (5'-3').

\begin{tabular}{ll}
\hline Primer name & \multicolumn{1}{c}{ Primer sequence } \\
\hline miR-510-RT & 5'-GTCGTATCCAGTGCAGGGTCCGAGGTATTCGCACTGGATACGACGTGATT-3' \\
miR-10a-RT & 5'-GTCGTATCCAGTGCAGGGTCCGAGGTATTCGCACTGGATACGACCACAAA-3' \\
U6-RT & 5'-CGCTTCACGAATTTGCGTGTCAT-3' \\
U6-F & 5'-GCTTCGGCAGCACATATACTAAAAT-3' \\
U6-R & 5'-CGCTTCACGAATTTGCGTGTCAT-3' \\
miR-510-F & 5'-TACTCAGGAGAGTGGCAATCA-3' \\
miR-10a-F & 5'-TACCCTGTAGATCCGAATTTG-3' \\
Universal reverse primer & 5'-GTGCAGGGTCCGAGGT-3'
\end{tabular}

F, forward primer; R, reverse primer; RT, reverse transcription primer.

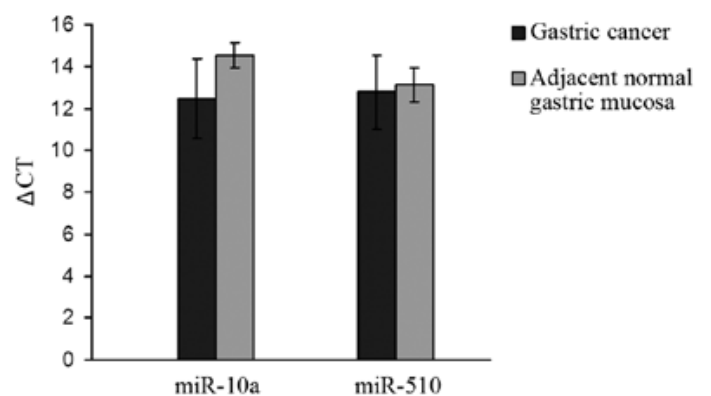

Figure 1. Relative expression of miR-10a and miR-510 in gastric cancer compared to adjacent normal gastric mucosa (higher $\Delta \mathrm{CT}$ represents a lower miRNA expression level). Expression of miR-10a in gastric cancer was higher than that in adjacent normal gastric mucosa $(\mathrm{P}<0.05)$.

\section{Results}

Results of the miRNA array. Considering the expression level of miRNAs in primary gastric cancer as baseline, there were 76 upregulated and 106 downregulated miRNAs associated with lymph node metastasis, among which four statistically significant miRNAs were included: three downregulated (miR24-1*, miR-510 and miR-1284) and one upregulated miRNA (miR-10a) (Table III).

Differentially expressed miRNAs in primary gastric tumors. We examined the two most obviously changed miRNAs (miR-10a and miR-510) in 33 cases of primary gastric cancer and corresponding normal tissue $(\mathrm{N})$. Each detected miRNA exhibited a good amplification curve; the melting curve had a single peak. The results suggested that the products of the real-time PCR were specific, and the experimental results were reliable.

Expression levels of miR-10a and miR-510 were measured in 33 cases of primary gastric cancer and its corresponding normal tissue. The average $\Delta \mathrm{Ct}$ (9) values of the tumor tissue were $12.48 \pm 1.91$ and $12.78 \pm 1.76$, respectively. The average $\Delta \mathrm{Ct}$ values of the corresponding adjacent normal tissue were $14.52 \pm 0.60$ and $13.11 \pm 0.80$, respectively. The expression of miR-10a was higher in gastric cancer than that in the adjacent normal mucosa $(\mathrm{P}<0.05)$ (Fig. 1).
Table III. Differentially expressed miRNAs detected by miRNA array.

\begin{tabular}{lcccc}
\hline & Fold change & P-value & \multicolumn{2}{c}{ Normalization } \\
\cline { 4 - 5 } miRNA & LN/T & LN/T & T & LN \\
\hline hsa-miR-24-1* $^{*} 0.4831$ & 0.0401 & 0.071 & 0.0343 \\
hsa-miR-510 & 0.3999 & 0.0069 & 0.0828 & 0.0331 \\
hsa-miR-1284 & 0.5716 & 0.0099 & 0.2366 & 0.1352 \\
hsa-miR-10a & 2.3929 & 0.0203 & 0.0401 & 0.096 \\
\hline
\end{tabular}

LN, lymph node metastasis tissue; T, tumor tissue.

Clinicopathologic characteristics. We investigated the relationship between expression of miR-10a and miR-510 and each of six clinicopathological characteristics (patient age, gender, tumor differentiation, major tumor histological classification, lymphatic invasion and lymph node metastasis). The results showed that only miR-10a expression was significantly related to node metastasis $(\mathrm{P}=0.047)$; this measure was not related to the state of lymphatic invasion $(\mathrm{P}=0.169)$ (Fig. 2 and Table IV).

Expression of miR-10a in the cell lines. The expression of miR-10a varied in all four cell lines. The expression of miR-10a in the MKN28, SGC7901 and AGS cell lines was 15-fold, 11.7-fold and 10.1-fold higher than that in the GES-1 cell line (Fig. 3). As MKN28 and SGC7901 were established from lymph node metastasis, the expression of miR-10a was higher in these two metastasis original cell lines than that in the AGS cell line which was established from a primary tumor.

Bioinformatic analysis of target genes for miR-10a. Potential target genes of miR-10a were predicted using miRGen; the corresponding results from the PicTar and TargetScanS databases were chosen as candidate targets; 118 potential target genes were obtained. KEGG analysis showed that the predicted target genes involved many signal pathways involved in tumorigenesis and tumor metastasis, such as the Wnt signal 
Table IV. Clinicopathological characteristics of the 33 cases of gastric cancer.

\begin{tabular}{|c|c|c|c|}
\hline \multirow[b]{2}{*}{ Variable } & \multirow[b]{2}{*}{ Cases, n (\%) } & \multicolumn{2}{|c|}{ P-value } \\
\hline & & miR-10a & $\operatorname{miR}-510$ \\
\hline Gender & & 0.861 & 0.918 \\
\hline Male & $28(84.8)$ & & \\
\hline Female & $5(15.2)$ & & \\
\hline Age (year) & & 0.874 & 0.280 \\
\hline$<60$ & $9(27.3)$ & & \\
\hline$\geq 60$ & $24(72.7)$ & & \\
\hline Types & & 0.978 & 0.691 \\
\hline Adenocarcinoma & $23(69.7)$ & & \\
\hline Tubular adenocarcinoma & $8(24.2)$ & & \\
\hline Signet ring cell carcinoma & $2(6.1)$ & & \\
\hline Differentiation & & 0.979 & 0.897 \\
\hline Moderately differentiated (II) & $10(30.3)$ & & \\
\hline Poorly differentiated (III) & $21(63.6)$ & & \\
\hline No differentiation (IV) & $2(6.1)$ & & \\
\hline Lymphatic invasion & & 0.169 & 0.365 \\
\hline Yes & $18(54.4)$ & & \\
\hline No & $15(45.5)$ & & \\
\hline Lymph node metastasis & & 0.047 & 0.204 \\
\hline Yes & $13(39.4)$ & & \\
\hline No & $20(60.6)$ & & \\
\hline
\end{tabular}
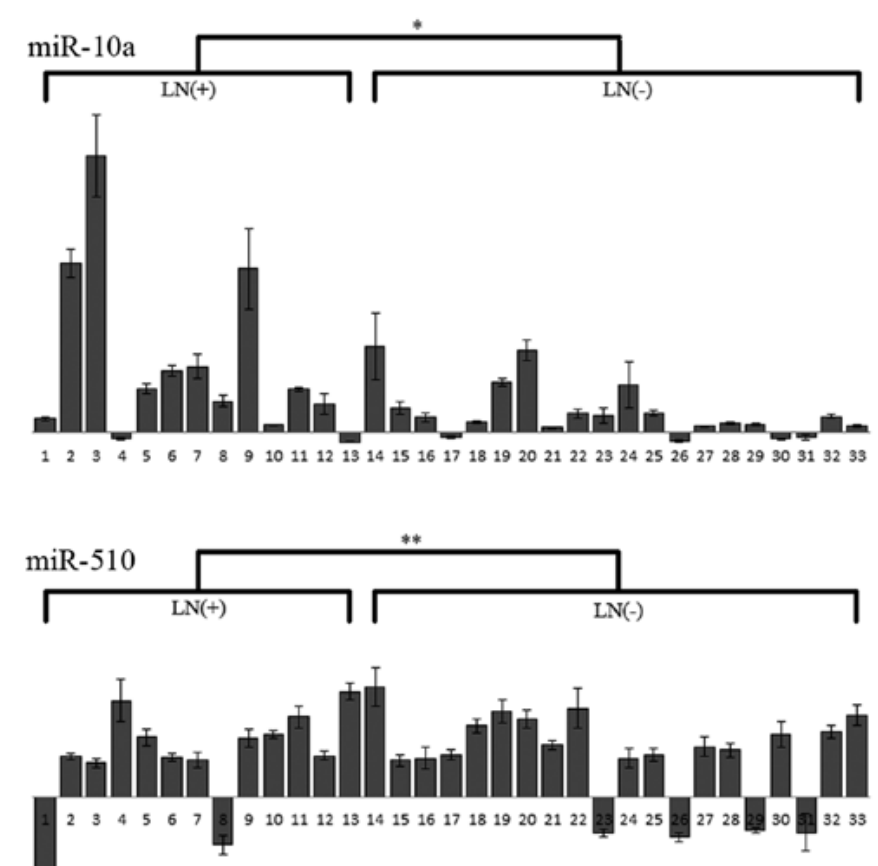

Figure 2. Expression of miR-10a and miR-510 in 33 primary gastric cancer tissues is demonstrated, respectively. $\mathrm{LN}(+) /(-)$ : patients with/without lymph node metastasis; ${ }^{*} \mathrm{P}=0.047 ;{ }^{* *} \mathrm{P}=0.204$.

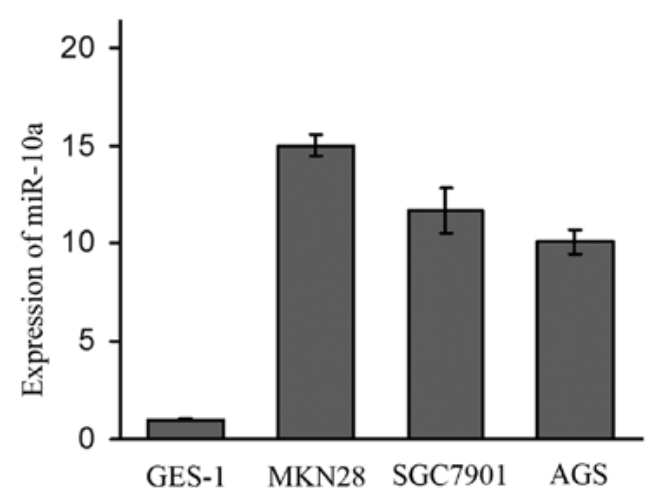

Figure 3. Expression of miR-10a, detected by real-time PCR, in MKN28, SGC7901 and AGS cell lines was 15-fold, 11.7-fold and 10.1-fold higher than that in the GES-1 cell line. Expression of miR-10a was higher in the two metastasis original cell lines (MKN28, SGC7901) than that in the AGS cell line which was established from a primary tumor.

pathway and that initiated by cell adhesion molecules (CAMs) (Table V).

\section{Discussion}

Few research efforts to date have examined the lymph node metastasis of gastric cancer using high-throughput methods $(10,11)$. These publications have focused mainly on the 
Table V. The most significant 5 pathways identified in the functional analysis of the miR-10a target genes.

\begin{tabular}{lll}
\hline Pathway & \multicolumn{1}{c}{ Gene } & P-value \\
\hline Wnt signaling pathway & BTRC, NFAT5, DVL3, CTNNBIP1, CAMK2G & 0.000013 \\
ErbB signaling pathway & SHC1, CRK, CAMK2G & 0.00069 \\
Focal adhesion & SHC1, ACTG1, CRK, FLT1 & 0.00074 \\
Cell adhesion molecules & NFASC, SDC1, CADM1 & 0.0024 \\
Notch signaling pathway & NCOR2, DVL3 & 0.0039 \\
\hline
\end{tabular}

genomic and proteomic differences between primary gastric cancer patients with metastasis and those without metastasis. Samples taken from different parts of the same person may help to avoid false-positive results on the miRNA array (caused by background differences among genes). In order to improve the specificity of the chip-based results, we chose to investigate primary gastric cancer and paired lymph node metastasis sites in the same patient. As the ratio of lymph node metastasis increases with the increased depth of invasion $(12,13)$, the specimens selected in the study all displayed fullthickness invasion.

The results obtained identified four differentially expressed miRNAs. miR-10a was upregulated while miR-24-1*, miR-510 and miR-1284 were downregulated in lymph node metastatic sites as compared to the primary gastric cancer. We hypothesized that if some relationship exists between the level of miRNA expression in the primary tumor sites and metastasis, it is quite possible that miRNAs contribute to the process of gastric cancer lymph node metastasis and may have the ability to serve as indicators in lymph node metastasis detection. In order to prove the hypothesis, we investigated the expression level of miR-10a and miR-510, which were the most obviously changed in the miRNA array, in the gastric cancer primary sites of 33 patients. The results showed that the expression level of miR-10a was affected by the existence of lymphatic metastasis, while there was no obvious relationship with the state of lymphatic invasion. Furthermore, miR-10a was upregulated in gastric cancer at both the tissue and cell levels; more obvious differences were noted at the cell level. Noticeably, the expression of miR-10a in the two metastasis original cell lines (MKN28 and SGC7910) was higher than that of the AGS cell line which was established from a primary tumor. This find also indicated that miR-10a was closely associated with lymph node metastasis. To assess the possibility of using miR-10a as a biomarker in lymph node metastasis, further research with more samples is needed.

miR-10a belongs to the miR-10 family; the other member is miR-10b. These two members differ from each other in only one base. Recently, several reports found that miR-10 expression was aberrantly increased in several types of cancers (14-17), upregulated in glioma, liver cancer, colon cancer, melanoma and breast cancer; downregulated in acute or chronic lymphocytic leukemia and head and neck squamouscell carcinoma. However, studies concerning the function of miR-10 are still lacking. Fortunately, miR-10 was proven to play a pivotal role in tumor invasion and metastasis in these studies. Ma et al (18) found that miR-10b was significantly correlated with breast tumor cell metastasis. They also demonstrated the ability of grafted tumors in mice to invade locally and transfer to distant sites and this was associated with upregulated expression of miR-10b in breast cancer cells lacking metastatic ability. In subsequent studies, the group effectively inhibited the distant metastasis of grafted mouse tumors using miR-10b antagomirs (chemically modified anti-miRNA oligonucleotides) (19). These results stimulated related research. Weiss et al (20) found that miR-10a was highly expressed in pancreatic cancer cell lines with high metastatic ability. Experimental manipulation of miR-10a expression altered the ability of pancreatic cancer cells to metastasize and invade. This pattern was also observed in zebrafish transplanted with pancreatic cancer. In gastric cancer, research on miR-10 is still limited to describing changes in miR-10 expression, with no elucidation of the detailed mechanisms. Li et al (21) found a seven-miRNA signature in gastric cancer (including miR-10b), which had a close relationship with disease-free survival and overall survival rate. Another study excluded a role for miR-10 family members (22). Although there is no report on whether miR-10 contributes to the mechanism of gastric cancer metastasis, the relationship of miR-10a to gastric cancer lymph node metastasis demonstrated in our research provides promising evidence for subsequent research.

miRNAs function post-transcriptionally during biological processes; therefore, definitive identification of miRNA target genes is essential. For this reason, we used bioinformatic methods to predict miR-10a target genes, and then analyzed the possible mechanisms of its role in gastric tumorigenesis and metastasis. We chose the most commonly used target genepredicting databases, PicTar (23) and TargetScanS (24), which are available on the miRGen website (25). To narrow the scope of this hunt for target genes and improve the specificity of the prediction, we chose the results common to both databases and subjected these to functional analysis. The analysis of KEGG (26) metabolic pathways showed that these predicted targets took part in many pathways involved in tumorigenesis, invasion and metastasis. For example, the Wnt signaling pathway plays an important role in organism development and gastrointestinal tumorigenesis (27), and cell adhesion molecules (CAMs) localized at the cell surface take part in intercellular and extracellular interactions.

These findings strongly suggest that miR-10a is closely correlated with gastric tumorigenesis and metastasis. A possible mechanism may be that it functions through interaction with target genes involved in tumorigenesis- and metastasis-related pathways. 


\section{Acknowledgements}

This study is supported by the Key Project of the Chinese Ministry of Education (no. 108015). We thank other members of our department for their valuable comments and help.

\section{References}

1. Wightman B, Ha I and Ruvkun G: Posttranscriptional regulation of the heterochronic gene lin-14 by lin- 4 mediates temporal pattern formation in C. elegans. Cell 75: 855-862, 1993.

2. Lee RC,Feinbaum RL and Ambros V: The C.elegans heterochronic gene lin-4 encodes small RNAs with antisense complementarity to lin-14. Cell 75: 843-854, 1993.

3. Bartel DP: MicroRNAs: genomics, biogenesis, mechanism, and function. Cell 116: 281-297, 2004.

4. Calin GA and Croce CM: MicroRNA signatures in human cancers. Nat Rev Cancer 6: 857-866, 2006.

5. Esquela-Kerscher A and Slack FJ: Oncomirs-microRNAs with a role in cancer. Nat Rev Cancer 6: 259-269, 2006.

6. Huang Q, Gumireddy K, Schrier M, et al: The microRNAs miR-373 and miR-520c promote tumor invasion and metastasis. Nat Cell Biol 10: 202-210, 2008.

7. Ma L and Weinberg RA: MicroRNAs in malignant progression. Cell Cycle 7: 570-572, 2008.

8. Chen C, Ridzon DA, Broomer AJ, et al: Real-time quantification of microRNAs by stem-loop RT-PCR. Nucleic Acids Res 33: e179, 2005.

9. Livak KJ and Schmittgen TD: Analysis of relative gene expression data using real-time quantitative PCR and the 2[-Delta Delta C(T)] method. Methods 25: 402-408, 2001.

10. Wang L, Zhu JS, Song MQ, et al: Comparison of gene expression profiles between primary tumor and metastatic lesions in gastric cancer patients using laser microdissection and cDNA microarray. World J Gastroenterol 12: 6949-6954, 2006.

11. Mori M, Mimori K, Yoshikawa Y, et al: Analysis of the geneexpression profile regarding the progression of human gastric carcinoma. Surgery 131 (Suppl 1): S39-S47, 2002.

12. Ushijima T and Sasako M: Focus on gastric cancer. Cancer Cell 5: $121-125,2004$.

13. Onate-Ocana LF, Aiello-Crocifoglio V, MondragonSanchez R, et al: Survival benefit of D2 lympadenectomy in patients with gastric adenocarcinoma. Ann Surg Oncol 7: 210-217, 2000 .
14. Agirre X, Jimenez-Velasco A, San Jose-Eneriz E, et al: Downregulation of hsa-miR-10a in chronic myeloid leukemia CD34 ${ }^{+}$ cells increase USF2-mediated cell growth. Mol Cancer Res 6: 1830-1840, 2008.

15. Zhang L, Huang J, Yang N, et al: MicroRNAs exhibit high frequency genomic alterations in human cancer. Proc Natl Acad Sci USA 103: 9136-9141, 2006.

16. Jongen-Lavrencic M, Sun SM, Dijkstra MK, et al: MicroRNA expression profiling in relation to the genetic heterogeneity of acute myeloid leukemia. Blood 111: 5078-5085, 2008.

17. Varnholt H, Drebber U, Schulze F, et al: MicroRNA gene expression profile of hepatitis $\mathrm{C}$ virus-associated hepatocellular carcinoma. Hepatology 47: 1223-1232, 2008.

18. Ma L, Teruya-Feldstein J and Weinberg RA: Tumor invasion and metastasis initiated by microRNA-10b in breast cancer. Nature 449: 682-688, 2007.

19. Ma L, Reinhardt F, Weinberg RA, et al: Therapeutic silencing of miR-10b inhibits metastasis in a mouse mammary tumor model. Nat Biotechnol 28: 341-347, 2010.

20. Weiss FU, Margues IJ, Woltering JM, et al: Retinoic acid receptor antagonists inhibit miR-10a expression and block metastatic behavior of pancreatic cancer. Gastroenterology 137: 2136-2145, 2009.

21. Li X, Zhang Y, Fan D, et al: Survival prediction of gastric cancer by a seven-microRNA signature. Gut 59: 579-585, 2010

22. Ueda T, Volinia S, Okumura H, et al: Relation between microRNA expression and progression and prognosis of gastric cancer: a microRNA expression analysis. Lancet Oncol 11: 136-146, 2010.

23. Krek A, Grun D, Poy MN, et al: Combinatorial microRNA target predictions. Nat Genet 37: 495-500, 2005.

24. Lewis BP, Burge CB and Bartel DP: Conserved seed pairing, often flanked by adenosines indicates that thousands of human genes are microRNA targets. Cell 120: 15-20, 2005.

25. Megraw M, Sethupathy P, Corda B, et al: miRGen: a database for the study of animal microRNA genomic organization and function. Nucleic Acids Res 35: D149-D155, 2007.

26. Kanehisa M and Goto S: KEGG: Kyoto encyclopedia of genes and genomes. Nucleic Acids Res 28: 27-30, 2000.

27. Polakis P: Wnt signaling and cancer. Genes Dev 14: 1837-1851, 2000. 